Meta

Journal des tradlucteurs

Translators' Journal

\title{
Traduction et développement de la terminologie française dans le domaine des affaires
}

\section{Zélie Guével}

Volume 35, numéro 1, mars 1990

Actes du colloque international « La traduction proligère »

URI : https://id.erudit.org/iderudit/002981ar

DOI : https://doi.org/10.7202/002981ar

Aller au sommaire du numéro

Éditeur(s)

Les Presses de l'Université de Montréal

ISSN

0026-0452 (imprimé)

1492-1421 (numérique)

Découvrir la revue

Citer cet article

Guével, Z. (1990). Traduction et développement de la terminologie française dans le domaine des affaires. Meta, 35(1), 154-161.

https://doi.org/10.7202/002981ar d'utilisation que vous pouvez consulter en ligne.

https://apropos.erudit.org/fr/usagers/politique-dutilisation/ 


\title{
TRADUCTION ET DÉVELOPPEMENT DE LA TERMINOLOGIE FRANÇAISE DANS LE DOMAINE DES AFFAIRES
}

\author{
ZÉLIE GUÉVEL \\ Ordre des comptables agréés du Québec, Montréal, Canada
}

La pratique de la traduction - de l'anglais au français - met constamment en évidence des besoins terminologiques; la réponse à ces besoins constitue, à mon avis, la contribution la plus remarquable de la profession de traducteur au Québec.

Je me propose de rendre compte ici de cette contribution en ce qui concerne le domaine des affaires et, plus particulièrement, du travail réalisé en terminologie comptable de langue française au cours des dernières décennies. Ma présentation comportera une analyse de l'évolution du vocabulaire des états financiers publiés durant la même période, évolution qui témoigne de la réussite du travail d'aménagement linguistique entrepris par la profession comptable. Je terminerai par un survol des travaux terminologiques menés récemment par divers organismes canadiens.

\section{LA TRADUCTION DANS LE DOMAINE DES AFFAIRES}

Pour commencer, il n'est sans doute pas inutile de préciser ce que l'on entend par le mot affaires et d'évoquer, à partir d'une expérience concrète, ce que recouvre la traduction dans ce domaine (parfois appelée traduction commerciale), ce qui permettra de mieux comprendre comment se manifestent les besoins terminologiques.

Le mot «affaires» désigne des activités qu'il n'est pas aisé de circonscrire. Si l'on se réfère au sens que revêt ce mot dans l'expression «droit des affaires» (nouvelle orientation du droit où l'entreprise constitue un élément central), on peut définir le domaine des affaires comme un champ d'application de l'économie, du point de vue de l'entreprise. Ce domaine regroupe les multiples activités économiques de l'entreprise, en particulier celles qui ont trait au commerce, aux finances et à la gestion'.

Dans le domaine des affaires, les textes à traduire présentent divers degrés de complexité, selon la spécialisation de l'organisme demandeur (service commercial d'une entreprise, institution financière, organisme de réglementation, etc.) et la nature du message. En fait, la variété caractérise nos tâches de traduction à plusieurs égards, même dans un service de traduction spécialisé comme celui qui servira ici d'exemple à mon propos, soit le service de traduction d'un organisme provincial et de l'organisme national qui représentent la profession comptable au Canada.

Dans un tel service, les textes à traduire peuvent traiter des méthodes en usage dans une diversité de secteurs, comme les mines, le pétrole et le gaz naturel, les assurances, les banques, les entreprises agricoles, les organismes de capital de risque ou les organismes sans but lucratif. Par ailleurs, la comptabilité dans les grandes entreprises fait appel à des techniques complexes, qu'il s'agisse - par exemple - de rendre compte des regroupements, des transactions effectuées en devises étrangères, des placements de portefeuille ou encore des coûts des régimes de retraite. La profession comptable établit aussi les normes concernant la vérification des états financiers et les services connexes offerts par l'expert-comptable, travaux qui font appel, entre autres, aux méthodes statistiques et aux

Meta, XXXV, 1,1990 
techniques informatiques. Depuis quelques années, les travaux de recherche et les prises de position publiées par la profession comptable concernent également le secteur public. Enfin, à la description des méthodes et techniques s'ajoutent des considérations théoriques sur les principes; par exemple, on s'est attaché récemment à redéfinir les fondements conceptuels des états financiers.

La diversité touche également la nature des textes. Outre les monographies, qui rendent compte de recherches spécialisées, et les normes (dans les différentes étapes de leur élaboration), le service auquel je fais référence assure la traduction des cours de formation continue et des examens de la profession, de rapports, brochures et lettres et autres types de documents, ainsi que d'une revue professionnelle - qui apporte chaque mois de nouveaux thèmes en relation avec l'actualité dans le monde des affaires.

Ainsi, la traduction dans le domaine des affaires fait appel à des connaissances spécialisées en continuelle évolution. Dans le contexte canadien, une très grande partie des recherches menées par les organismes d'envergure nationale se fait en langue anglaise et, à tous les niveaux de communication, le traducteur demeure un chaînon essentiel. Le rôle d'intermédiaire assumé par le traducteur est d'autant plus important que son organisme s'adresse à une vaste section de la population et que les communications y sont primordiales - ce qui est le cas pour la profession comptable (dont les activités sont axées sur la communication d'informations, laquelle s'effectue tant par les mots que par les chiffres).

Pour assurer une communication efficace, il est fondamental que le traducteur veille à la justesse technique et linguistique des termes qu'il emploie (l'improvisation ne pouvant être de mise dans les langues de spécialité). Celle-ci doit être respectée, par exemple, à l'intérieur d'un même document dont la publication des divers chapitres et des nombreux changements qui y sont apportés s'échelonne sur des années (comme le recueil de normes que constitue le Manuel de l'Institut canadien des comptables agréés) mais aussi d'un document à l'autre (par exemple, textes des examens, normes et documents visant à vulgariser la recherche), de sorte que les mêmes réalités soient toujours désignées de la même manière. (Cette contrainte peut même se faire pesante dans les textes de normalisation.) En plus de bien connaître le vocabulaire des disciplines dont il traite, le traducteur travaillant dans le domaine des affaires est appelé bien souvent à désigner en français les concepts ou les méthodes nouvellement apparus. Il devient, en quelque sorte, un gestionnaire des langues de spécialité.

Le travail que j'ai évoqué est celui d'un service de traduction mis en place par la profession comptable du Québec, il y a vingt ans, et qui agit de concert avec un comité de terminologie française, parmi les premiers du genre, créé à la même période. Les réalisations de ce comité ainsi que la publication d'un dictionnaire, dans le sillage de la traduction, constituent un élément marquant de la contribution terminologique de la profession comptable, dont il sera question ci-après. Pour comprendre cette contribution, il faut tenir compte de la situation qui prévalait dans les années soixante et des problèmes particuliers que pose l'établissement d'une terminologie dans le domaine de la comptabilité au Québec.

\section{LA CONTRIBUTION TERMINOLOGIQUE DE LA PROFESSION COMPTABLE LA SITUATION DANS LES ANNÉES SOIXANTE}

En réalité, c'est du côté de l'enseignement que se sont manifestés d'abord les besoins, dès le début des années soixante, à l'École des Hautes Études Commerciales (H.É.C.) de Montréal et à l'Université Laval.

Quelle était alors la situation? Dans un article sur les activités terminologiques de la profession comptable au Canada (paru l'an dernier dans TermNet $N e w s^{2}$ ), on fait 
mention de la situation de déficit linguistique qui caractérisait les années soixante. «À l'époque, précise-t-on, la plupart des francophones qui s'orientaient vers la profession comptable avaient fait leurs études spécialisées ou leur stage de formation en anglais et se trouvaient dépourvus d'une bonne partie du vocabulaire technique français de leur profession.» Dans un article publié dans l'Actualité terminologique - qui reprend une communication présentée au Séminaire international sur le français, langue des affaires (qui s'est tenu en 1979 à Sophia Antipolis ${ }^{3}$ ), on mentionne que l'enseignement de la comptabilité à l'école des H.É.C. de Montréal s'était surtout donné, jusqu'aux années quarante, avec des ouvrages importés de France. Cependant, indique-t-on dans l'article, «la brisure causée par le conflit mondial oriente davantage le Québec vers les États-Unis. Les manuels américains (...) deviennent les ouvrages de référence d'un enseignement que l'on continue de dispenser en français. Cette influence américaine accentue le besoin d'un vocabulaire comptable en français déjà nécessaire à cause de la force prépondérante du milieu anglo-canadien».

La tâche n'est pas facile, comme le constatent, en 1960, les auteurs de Termes comptables, le premier lexique anglais-français établi par la profession (destiné à accompagner le dictionnaire unilingue de l'Institut canadien des comptables agrés, Terminology for Accountants):

Pour nous qui vivons dans une province bilingue, le besoin d'une terminologie française se fait sentir de façon de plus en plus pressante (...)

Alors que d'autres sciences peuvent faire appel à des ouvrages et des manuels d'origine française, les divergences considérables entre nos méthodes comptables et celles qui sont employées en France nous privent du contact des auteurs dans le domaine de la comptabilité. Par suite de cette rupture, nous sommes pratiquement laissés à nos propres moyens en ce qui concerne la formation et l'emploi des termes comptables de langue française qui correspondent à ces méthodes.

L'affirmation est plus nuancée dans la seconde édition (1963), où les mêmes auteurs signalent que certains des changements résultent de [leur] intention de tenir compte davantage de l'usage européen (...) en raison des relations économiques sans cesse croissantes entre l' Europe et l'Amérique.

En fait, le cadre juridique dans lequel s'inscrit la comptabilité est très différent en Europe, où un plan comptable est mis en œuvre dès 1942. D'abord appliqué sur une base volontaire, le plan comptable devient obligatoire pour les sociétés nationalisées françaises en 1947; cette obligation s"étend aux sociétés privées d'une certaine importance, à partir de 1964. Le plan comptable constitue un cadre rigide; la terminologie est uniformisée et se présente comme une nomenclature chiffrée. Dans la comptabilité nord-américaine, le travail de normalisation porte sur les principes généraux et une plus grande liberté demeure quant à la forme.

\section{LE COMITÉ DE TERMINOLOGIE FRANÇAISE}

La prise de conscience des problèmes que pose la comptabilité d'expression française par les instances dirigeantes de la profession comptable au Québec (Ordre des comptables agréés du Québec) aboutit à la création, en 1964, d'un comité de terminologie française et, quelques années plus tard (en 1969), d'un service de traduction. Le Comité de terminologie française a pour mandat de travailler à répandre le bon usage parmi les membres de la profession, les étudiants et le large public du monde des affaires. Pour réaliser ce mandat, il entreprend, à partir de 1966, la publication de bulletins, Terminologie comptable, qui traitent de problèmes terminologiques ponctuels. Pour préciser les choses, mentionnons que le Comité de terminologie est constitué d'universitaires et de praticiens 
agissant comme bénévoles, auquel s'est joint le terminologue Robert Dubuc dès les premières années. L'administration et le secrétariat du comité relèvent du Service de traduction. Depuis 1966, une soixantaine de bulletins ont été publiés, dont une première série a été refondue dans une plaquette, en 1983.

Au fil des années, le Comité s'est défini un corps de principes ${ }^{4}$. Le premier consiste en un alignement sur l'usage européen, avec les finalités suivantes : éviter les dangers du ghetto linguistique, assurer [au] français une plus grande force de résistance à l'anglicisation et donner [à la] langue de spécialité une plus grande cohésion interne. À ce principe s'ajoutent trois autres: résistance au calque de l'anglais; en cas de nécessité, dérogation à l'usage international pour assurer le respect des particularités locales et, dans ce dernier cas, application d'un principe du seuil de compréhension (adoption de termes qui ne posent pas d'ambiguïté pour un francophone connaissant un tant soit peu le domaine).

De façon générale, les articles des bulletins fournissent une description des notions traitées, précisent la problématique - laquelle découle le plus souvent d'un contexte de traduction - , font le point sur les divers choix et justifient la solution retenue. Dans la méthodologie appliquée par le Comité, la consultation des membres est considérée comme essentielle: une procédure de consultation systématique de correspondants a été mise en place en 1979. Le nombre de ceux-ci s'est élargi avec le temps et des représentants de la profession d'expert-comptable en Belgique et en France font maintenant partie des personnes consultées. Les bulletins sont distribués à tous les membres de la profession et aux autres personnes intéressées qui en font la demande. Bien entendu, la terminologie établie par le Comité est utilisée dans tous les textes qui passent par le service de traduction, en particulier dans le recueil de normes déjà mentionné (Manuel de l'Institut canadien des comptables agréés).

\section{ÉVOLUTION DU VOCABULAIRE COMPTABLE ${ }^{5}$}

La terminologie des états financiers constitue le premier noyau de la recherche menée par le Comité de terminologie française. Il était donc intéressant de voir comment avait évolué, sur une vingtaine d'années (de 1965 à 1984), la terminologie présentée dans les états financiers des grandes entreprises et si cette évolution faisait écho au travail terminologique mené par la profession. Pour mener cette étude, j'ai retenu vingt grandes entreprises industrielles ou commerciales dont le siège social se trouve au Québec et j'ai relevé, dans les états financiers annuels publiés par ces entreprises, la manière dont avaient été rendues une quinzaine de notions. Les états financiers fournissent un cadre systématique grâce auquel il m'a été possible de dresser pour chaque notion, année après année, la liste des termes utilisés et le nombre total d'attestations, puis de procéder à une représentation graphique visant à mettre en relief l'évolution comparative des termes. L'étude a porté sur les termes préconisés dans les bulletins de terminologie et qui sont aussi repris dans le recueil de normes (à l'exception de deux, qui se trouvent seulement dans le recueil de normes). Dans tous les cas, il s'agissait de notions qui avaient posé des problèmes terminologiques. Ainsi, en résumé :

- plusieurs termes visaient un alignement sur l'usage européen :

- stocks (pour remplacer le calque inventaires, au sens de produits disponibles);

- exercice (pour désigner la période - généralement de douze mois - à la fín de laquelle on ferme les comptes d'une entreprise, qui était bien souvent appelée année, année budgétaire, voire année fiscale);

- comptes débiteurs et comptes créditeurs et les spécifiques correspondants, comptes clients et comptes fournisseurs (pour remplacer comptes à payer et comptes à recevoir); 
- produits et charges (pour remplacer, dans l'état des résultats, les revenus et les dépenses;

- charges à payer (en remplacement de dettes courues - l'adjectif couru étant employé dans un autre contexte en comptabilité européenne - pour rendre accrued liabilities);

- chiffre d'affaires (en remplacement de ventes);

- bénéfice (en remplacement de revenu, pour réduire la synonymie et régler les hésitations découlant de l'emploi du pluriel en anglais - cf.earnings);

d'autres étaient plutôt forgés pour désigner des particularités de la comptabilité nordaméricaine:

- actif à court terme et passif à court terme (en remplacement de disponibilités et exigibilités; correction technique);

- état de l'évolution de la situation financière; état des résultats; état des bénéfices non répartis (en remplacement d'une série de synonymes);

- notes complémentaires, notes afférentes aux états financiers (pour corriger des tournures boiteuses comme notes aux (des) états financiers).

L'incidence sur les états financiers des choix terminologiques de la profession peut s'analyser sous trois aspects: les résultats atteints à la fin de la période examinée (1984), l'évolution par rapport à une situation de départ (en 1965) et le rythme des changements.

À propos des résultats obtenus pour 1984, on constate :

- un succès relatif en matière d'implantation terminologique pour les cas suivants (en ordré décroissant) :

- les 20 entreprises observées utilisent les termes stocks, exercice, actif à court terme, passif à court terme et état de l'évolution de la situation financière;

- 18 utilisent état des résultats (qui a pour concurrents : état du bénéfice et état du revenu); 18 utilisent bénéfice (les deux autres utilisent le même terme au pluriel);

- 17 utilisent état des bénéfices non répartis (concurrents : état des bénéfices réinvestis et état des revenus non répartis);

- 16 utilisent notes complémentaires, notes afférentes aux états financiers, notes accompagnant les états financiers;

- 15 utilisent comptes débiteurs (ou le spécifique compte clients) et comptes créditeurs (ou le spécifique compte fournisseurs);

que d'autres termes n'ont pas encore fait la preuve de leur viabilité :

- 6 entreprises utilisent charges ( 6 autres utilisent dépenses);

- 4 entreprises utilisent charges à payer (7 autres utilisent frais courus);

- 2 entreprises utilisent chiffre d'affaires (15 utilisent ventes);

- 2 entreprises utilisent produits (15 utilisent revenus).

De façon générale, nous avons constaté que le facteur temps est un élément essentiel dans l'implantation terminologique. Ainsi, quatre des cinq termes dont l'usage fait l'unanimité ont été proposés entre 1965 et 1972 et le cinquième, en 1974. Sur les quatre termes qui semblent moins bien passer, trois ont été proposés en 1980 et 1981.

Si l'on observe l'évolution des termes tout au long de la période, on constate une progression pour chacun des termes préconisés. Exercice, par exemple, est employé par 13 entreprises en 1965; l'unanimité à l'égard de son emploi se fait en 1975. D'autres termes inconnus ou rarement utilisés avant d'être recommandés sont adoptés rapidement (actif à court terme, passif à court terme, état de l'évolution de la situation financière, comptes créditeurs, etc.). Quant au rythme des changements, on remarque qu'un terme nouveau ou peu connu est adopté presque tous les ans (11 termes en 14 ans). 
Nous avons comparé ces résultats qui, rappelons-le, concernent un usage écrit (celui des rapports annuels de la grande entreprise) à ceux d'un sondage mené par le Comité en 1983 dans lequel on demandait notamment aux comptables agréés d'indiquer, parmi les changements proposés par le Comité (soit une liste de termes), ceux qu'ils avaient retenus. Les résultats obtenus sont similaires. Les résultats du sondage ont été analysés dans un article de la revue professionnelle CAmagazine ${ }^{6}$, dont voici l'une des conclusions: On peut se risquer à en déduire que les comptables agréés ont une conscience assez aiguë des problèmes ou déficiences à l'origine de l'évolution accélérée de la terminologie comptable au Québec.

\section{AUTRES RÉALISATIONS DE LA PROFESSION COMPTABLE}

Mon étude portait sur les termes de base en comptabilité. Mais la contribution du Comité ne s'arrête pas à ces quelques termes. Plusieurs centaines de notions ont été traitées dans les bulletins. Outre la comptabilité générale (soit les intitulés des états financiers, les postes et les comptes, les méthodes et les systèmes), ces notions concernent le travail de vérification et l'organisation de la vie professionnelle, ainsi que la fiscalité, l'informatique, la gestion et la prise de décision et une variété de notions propres au commerce et à la vie des entreprises. Parmi les articles récents, signalons :

«Leverage» (effet de levier financier), où l'on propose des néologismes («rachat adossée pour leveraged management buyout; «bail adossé» pour leveraged lease); en «théorie comptable», qui fait le point sur plusieurs théories, dont la «théorie de l'intérêt de l'agent» (agency theory) et Goodwill, où l'on propose de remplacer achalandage par fonds commercial (entreprise individuelle) ou écart d'acquisition (consolidation)

Enfin, on ne pourrait passer sous silence l'importante réalisation que représente le Dictionnaire de la comptabilité et des disciplines connexes, dont la préparation de la troisième édition est amorcée. Réalisé par l'Institut canadien des comptables agréés, c'est - pour les deux premières éditions - l'œuvre d'un professeur de comptabilité et traducteur de manuels, le regretté Fernand Sylvain. Les deux collaborateurs principaux sont également des traducteurs. Après les deux éditions de Termes comptables, lexique bilingue (sans définitions) visant à accompagner Terminology for Accountants, on a ressenti le besoin d'avoir un ouvrage plus autonome, doté de définitions, avec une nomenclature élargie. Le Dictionnaire de la comptabilité a pour originalité de présenter une nomenclature anglaise, avec définitions et équivalents donnés en français. La première édition, qui paraît en 1977, contient déjà une nomenclature plus importante que celle de Terminology of Accountants (soit 1518 termes). La deuxième édition (1982) compte plus de cinq mille termes: l'intention de l'auteur est d'y englober tous les termes techniques que le comptable est susceptible d'utiliser dans l'exercice de la profession. Enfin, dans la deuxième édition, la vocation internationale de l'ouvrage se précise. L'auteur note dans la préface: L'objectif de cet ouvrage n'est pas d'uniformiser [la] terminologie [Canada, Belgique, France...] mais de faire connaître ce qui est en usage ailleurs de façon à ce que tous puissent saisir la portée des termes utilisés.

Grâce aux travaux terminologiques menés depuis plus de vingt ans, travail de réflexion et de diffusion, la profession comptable dispose aujourd'hui d'un vocabulaire solide. Les lacunes ont été comblées. Mais si le travail de rattrapage a été fait, il importe de rester vigilant car de nouveaux besoins se font jour constamment (ainsi, pour ne citer qu'un exemple, ces dernières années, la prolifération d'instruments financiers comme les contrats à terme, les options, les swaps de devises ou de taux d'intérêt - 
désignation qu'on cherche actuellement à revoir — oblige à définir une nouvelle terminologie).

AUTRES RÉALISATIONS TERMINOLOGIQUES DANS LE DOMAINE DES AFFAIRES AU CANADA

D'autres associations canadiennes cuvrant dans le monde des affaires se préoccupent aussi de leur terminologie et il semble bien que la prise de conscience se fasse plus vive en cette fin des années quatre-vingt. Ainsi :

- la Société des comptables en management du Canada publie depuis quelques années son bulletin Lexicom;

- l'Association canadienne des compagnies d'assurance de personnes a publié, en 1982, son Vocabulaire des rapports annuels;

- l'Institut canadien des actuaires dispose depuis quelques années d'un Lexique, c'est-à-dire d'une liste d'équivalences (qui, selon la préface, répond à un besoin de normalisation et propose à cette fin le vocabulaire le plus répandu sur le plan international);

- l'Institut canadien des valeurs mobilières a aussi mis au point son Lexique (avec définitions) destiné à aider les étudiants à se préparer aux examens de l'Institut;

- l'Association pour la gestion de la production et des stocks, en collaboration avec l'École des H.É.C. de Montréal et l'Office de la langue française, prépare actuellement un Vocabulaire (version préliminaire parue en 1988). Ce vocabulaire vise à fournir les équivalents français (avec définitions) des termes figurant dans le dictionnaire unilingue de l'Association. Il s'agit d'un important projet où l'on a prévu une collaboration avec la France et les autres pays francophones. Les responsables entendent ensuite obtenir la réalisation d'une version française des examens de l'association américaine de ce domaine (gestion de la production et des stocks);

- l'Institut des experts en évaluation d'entreprises ressent actuellement le besoin d'établir sa terminologie, en particulier pour des fins de formation.

Au chapitre des réalisations terminologiques dans le domaine des affaires, citons encore les récentes publications du Secrétariat d'État: Vocabulaire de la bourse et du placement et Vocabulaire de la banque, ainsi que le Lexique budgétaire, comptable et financier, paru en 1982. De son côté, l'Office de la langue française prépare notamment un vocabulaire de l'immobilier, un lexique général des assurances et un vocabulaire des techniques actuarielles.

L'activité traductionnelle au Canada a contribué pour beaucoup au développement des langues de spécialité, en particulier dans le domaine des affaires. Des principes et des méthodes d'aménagement ont aussi été dégagés. Parmi ceux-ci, la participation des usagers de la langue de spécialité est vraiment perçue comme essentielle. Pour la poursuite du travail, on ressent le besoin d'une concertation des efforts au sein de la francophonie, d'abord pour éviter la multiplication du travail (alors que la tâche est immense), mais surtout pour assurer une harmonie dans les usages, garante d'une efficacité dans les communications, qui sont appelées à s'internationaliser de plus en plus. Quant aux réalités déjà bien cernées, il semble qu'un regroupement des résultats, sous une forme commode d'accès, paraît de plus en plus souhaitable, ainsi que - peut-être - l'élaboration du vocabulaire de base de ce tronc commun que représente le domaine des affaires. 


\section{NOTES}

1. Il s'agit de la définition que j'ai proposée dans un mémoire de D.E.A., Recherche préliminaire sur la terminologie du français des affaires dans les dictionnaires thématiques, sous la direction de Pierre Lerat, Paris XIII, juin 1989, document dactylographié.

2. JOLY, Jean-François (1988): «Les activités terminologiques de la profession comptable au Canada», TermNet News, 2, pp. 36-41.

3. CôTÉ, Yves-Aubert (1980): «Aperçu sur l'effort québécois en terminologie comptable française», L'Actualité terminologique, pp. 67-68.

4. Ces principes sont expliqués dans les premières pages de l'ouvrage du Comité de terminologie française (1983) : Terminologie comptable, Ordre des comptables agrés du Québec. Cet ouvrage est une refonte de la première série de bulletins Terminologie comptable, qui ont été publiés de 1966 à 1982.

5. La présente section reprend sommairement les résultats d'un travail dirigé de maitrise, sous la direction d'André Clas, Évolution du vocabulaire comptable au Québec de 1965 à 1984 dans les états financiers de vingt entreprises, Université de Montréal, juin 1986, document dactylographié.

6. ARSENAULT, Murielle et Jean-François JOLY (1986): «Les comptables agréés du Québec et les changements terminologiques», CAmagazine, janvier, pp. 54-61. 